\title{
4D-STEM Pair Distribution Function Mapping of the Morphology and Structure of Amorphous Organic Materials
}

\author{
Xiaoke $\mathrm{Mu}^{1}$, Andrey Mazilkin ${ }^{1}$, C. Sprau ${ }^{2}$, A. Colsmann ${ }^{2}$ and Christian Kübel ${ }^{1,3,4^{*}}$ \\ 1. Institute for Nanotechnology (INT), Karlsruhe Institute of Technology (KIT), Eggenstein- \\ Leopoldshafen, Germany. \\ 2. Light Technology Institute, Karlsruhe Institute of Technology (KIT), Karlsruhe, Germany. \\ ${ }^{3 .}$ Helmholtz-Institute Ulm for Electrochemical Energy Storage (HIU), Karlsruhe Institute of Technology \\ (KIT), Ulm, Germany. \\ 4. Karlsruhe Nano Micro Facility (KNMF), Karlsruhe Institute of Technology (KIT), Eggenstein- \\ Leopoldshafen, Germany. \\ * Corresponding author: christian.kübel@kit.edu
}

Imaging organic materials at the nanoscale as well as analyzing their local atomic structure has been a long-time challenge because of the weak scattering from carbon-based materials, their amorphous structure and beam sensitive nature. Here, we propose a new low-dose approach for imaging the phase distribution and for analysing the local structure of amorphous or semi crystalline organic materials [1] based on scanning transmission electron diffraction (4D-STEM) pair distribution function analysis (PDF) [2].

We show that PDF analysis of electron diffraction [3] can be used to characterize the short- and mediumrange order in aperiodically packed organic molecules. It allows to decode the complex structural information encoded in the amorphous features of the diffraction pattern. For example, as shown in figure 1, the structural evolution due to electron beam damage can be monitored by comparing the PDFs with molecular dynamic (MD) modelling derived structures. This allows to understand the molecular packing and beam induced bond breakage. Moreover, we show that 4D-STEM-PDF (figure 2) does not only provide local structural information with a resolution of a few nanometers, but can also be used to image the phase distribution of organic composites. The distinct and thickness independent contrast is generated by utilizing the structural difference between the different types of molecules and taking advantage of the dose efficiency due to use of the full scattering signal. Therefore, this approach is particularly interesting for imaging unstained organic or polymer composites without distinct valence states for electron energy loss spectroscopy. In addition, we will also demonstrate that the 4D-STEM data can not only help us to optimise the conditions of convergence/scattering angle in conventional STEM-BF/DF to image the phase distribution of organic molecules with reduced dose, but also provides phase contrast for ultralow dose imaging.

We explore the possibilities of this new approach using [6,6]-phenyl C61-butyric acid methyl ester (PCBM) and poly(3-hexylthiophene-2,5-diyl) (P3HT) as the archetypical and best-investigated semiconductor blend used in organic solar cells and compare our phase distribution with virtual dark-field analysis and validate our approach by electron energy loss spectroscopy [4].

\section{References:}

[1] X Mu et al., Microscopy (2019) accepted.

[2] X Mu et al., Ultramicroscopy 168 (2016), p. 1. 
[3] X Mu et al., J. Appl. Crystallogr. 46 (2013), p. 1105.

[4] The authors thank Quantum Detector Ltd (UK) for sharing their Medipix3-S1 detector for the project. X Mu acknowledge Deutsche Forschungsgemeinschaft's (MU 4276/1-1) support for the development of STEM-PDF.

(a)

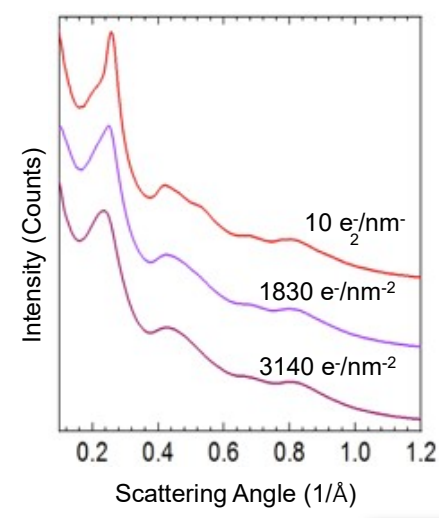

(b)

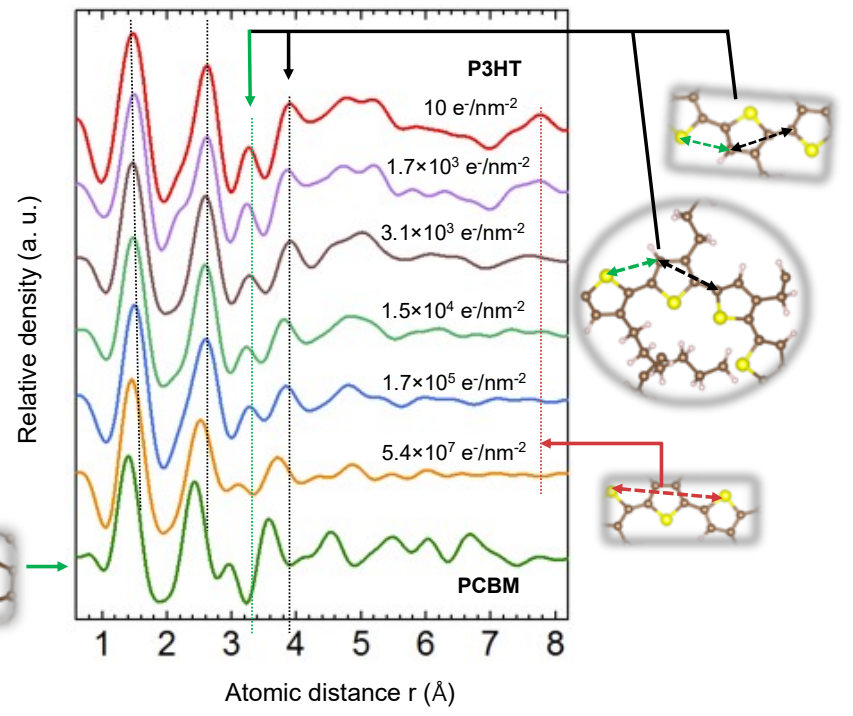

Figure 1. Beam induced structural changes in the neat P3HT film measured at $300 \mathrm{keV}$ acceleration voltage. (a) Azimuthally integrated diffraction profiles and (b) PDFs. The legend notes the dose. The top red and bottom green are PDFs of $\mathrm{P} 3 \mathrm{HT}$ and $\mathrm{PC}_{61} \mathrm{BM}$ for comparison. The atomic model for $\mathrm{P} 3 \mathrm{HT}$ was constructed by MD simulation.
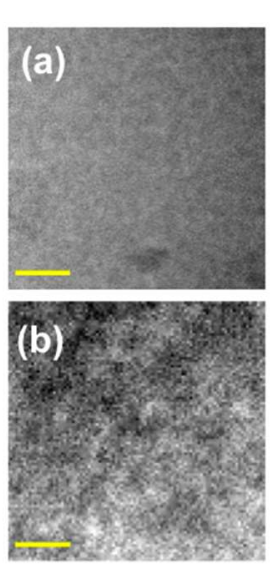
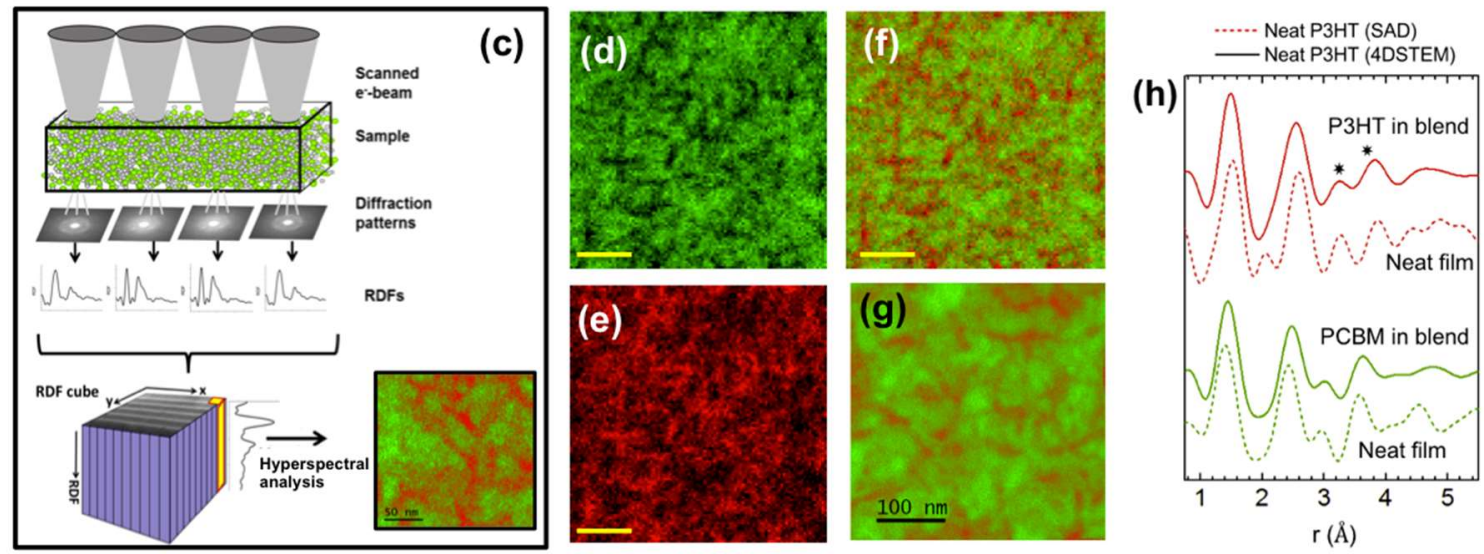

Figure 2. (a) A typical (HR)TEM bright field image of the blend film. (b) Virtual STEM HAADF image. (c) Procedure for STEM-PDF analysis. (d) PDF maps of PCBM and (e) the P3HT phase at the same location as in b. (f) RGB color mix of d (green) and e (red). (g) A typical EFTEM map of the blend film. (h) PDFs of the P3HT-rich (red solid line) and PCBM-rich (green solid line) phases of the blend extracted from the STEM-PDF cube. PDFs of the neat P3HT (red dashed line) and PCBM (green dashed line) films obtained by using the SAED microscope setup. All scale bars are $100 \mathrm{~nm}$. 